\title{
Bernstein Type Inequalities for Polar Derivative of Polynomial
}

\section{Barchand Chanam ${ }^{1}$}

${ }^{1}$ Department of Mathematics National Institute of Technology Manipur, Imphal, Manipur, India barchand_2004@yahoo.co.in

Article History: Received: 11 January 2021; Accepted: 27 February 2021; Published online: 5 April 2021

Abstract- If $p(z)$ is a polynomial of degree $n$ such that $p(z) \neq 0$ in $|z|<k, k \leq 1$, then Govil [ Proc. Nat. Acad. Sci., Vol. 50, pp. 50-52, 1980. ] proved

$$
\max _{|z|=1}\left|p^{\prime}(z)\right| \leq \frac{n}{1+k^{n}} \max _{|z|=1}|p(z)|
$$

provided $\left|p^{\prime}(z)\right|$ and $\left|q^{\prime}(z)\right|$ attain their maxima at the same point on the circle $|z|=1$, where $q(z)=z^{n} \overline{p\left(\frac{1}{\bar{z}}\right)}$.

Equality in the above inequality holds for $p(z)=z^{n}+k^{n}$.

In this paper, we extend the above inequality and an improved version of this into polar derivative of a polynomial.

Keywords - Polynomial, Polar Derivative of a polynomial, Inequalities, Maximum Modulus.

\section{Introduction}

It was for the first time, Bernstein $[10,11]$ investigated an upper bound for the maximum modulus of the first derivative of a complex polynomial on the unit circle in terms the maximum modulus of the polynomial on the same circle and proved the following famous result known as Bernstein's inequality that if $p(z)$ is a polynomial of degree $n$, then

$$
\max _{|z|=1}\left|p^{\prime}(z)\right| \leq n \max _{|z|=1}|p(z)| \text {. }
$$

Inequality (1.1) is best possible and equality occurs for $p(z)=\lambda z^{n}, \lambda \neq 0$, is any complex number.

If we restrict to the class of polynomials having no zero in $|z|<1$, then inequality (1.1) can be sharpened as

$$
\max _{|z|=1}\left|p^{\prime}(z)\right| \leq \frac{n}{2} \max _{|z|=1}|p(z)| .
$$

The result is sharp and equality holds in (1.2) for $p(z)=\alpha+\beta z^{n}$, where $|\alpha|=|\beta|$.

Inequality (1.2) was conjectured by Erdös and later proved by Lax [8].

Simple proofs of this theorem were later given by de-Bruijn [5], and Aziz and Mohammad [2].

It was asked by R.P. Boas that if $p(z)$ is a polynomial of degree $n$ not vanishing in $|z|<k, k>0$, then how large can

$$
\left\{\max _{|z|=1}\left|p^{\prime}(z)\right| / \max _{|z|=1}|p(z)|\right\} \text { be ? }
$$

A partial answer to this problem was given by Malik [9], who proved

Theorem A. If $p(z)$ is a polynomial of degree $n$ having no zero in the disc $|z|<k, k \geq 1$, then

$$
\max _{|z|=1}\left|p^{\prime}(z)\right| \leq \frac{n}{1+k} \max _{|z|=1}|p(z)|
$$


The result is best possible and equality holds for $p(z)=(z+k)^{n}$.

For the class of polynomials not vanishing in $|z|<k, k \leq 1$, the precise estimate for maximum of $\left|p^{\prime}(z)\right|$ on $|z|=1$, in general, does not seem to be easily obtainable.

For quite some time, it was believed that if $p(z) \neq 0$ in $|z|<k, k \leq 1$, then the inequality analogous to (1.4) should be

$$
\max _{|z|=1}\left|p^{\prime}(z)\right| \leq \frac{n}{1+k^{n}} \max _{|z|=1}|p(z)|
$$

till E.B. Saff gave the example $p(z)=\left(z-\frac{1}{2}\right)\left(z+\frac{1}{3}\right)$ to counter this belief.

Govil [6] obtained inequality (1.5) with extra condition. More precisely, he proved the following

Theorem B. If $p(z)$ is a polynomial of degree $n$ such that $p(z) \neq 0$ in $|z|<k, k \leq 1$, then

$$
\max _{|z|=1}\left|p^{\prime}(z)\right| \leq \frac{n}{1+k^{n}} \max _{|z|=1}|p(z)|,
$$

provided $\left|p^{\prime}(z)\right|$ and $\left|q^{\prime}(z)\right|$ attain their maxima at the same point on the circle $|z|=1$, where $q(z)=z^{n} \overline{p\left(\frac{1}{\bar{z}}\right)}$

Equality in (1.6) holds for $p(z)=z^{n}+k^{n}$.

Aziz and Rather [3] further improved the bound of (1.6) by involving $\min _{|z|=k}|p(z)|$.

Theorem C. If $p(z)$ is a polynomial of degree $n$ such that $p(z) \neq 0$ in $|z|<k, k \leq 1$, then

$$
\max _{|z|=1}\left|p^{\prime}(z)\right| \leq \frac{n}{1+k^{n}}\left\{\max _{|z|=1}|p(z)|-\min _{|z|=k}|p(z)|\right\} \text {, }
$$

provided $\left|p^{\prime}(z)\right|$ and $\left|q^{\prime}(z)\right|$ attain their maxima at the same point on the circle $|z|=1$, where $q(z)=z^{n} \overline{p\left(\frac{1}{\bar{z}}\right)}$.

As in Theorem B, equality in (1.6) occurs for $p(z)=z^{n}+k^{n}$.

Let $p(z)$ be a polynomial of degree $n$ and $\alpha$ be any real or complex number, the polar derivative of $p(z)$, denoted by $D_{\alpha} p(z)$, is defined as

$$
D_{\alpha} p(z)=n p(z)+(\alpha-z) p^{\prime}(z) \text {. }
$$

The polynomial $D_{\alpha} p(z)$ is of degree at most $n-1$ and it generalizes the ordinary derivative $p^{\prime}(z)$ of $p(z)$ in the sense that

$$
\lim _{\alpha \rightarrow \infty} \frac{D_{\alpha} p(z)}{\alpha}=p^{\prime}(z)
$$

It is of interest to extend ordinary derivative inequalities into polar derivative of a polynomial, for the later version is a generalization of the first. In this direction, Aziz and Shah [4] for the first time extended (1.1) to polar derivative by proving

Theorem D. If $p(z)$ is a polynomial of degree $n$ then for every real or complex number $\alpha$ with $|\alpha| \geq 1$,

$$
\max _{|z|=1}\left|D_{\alpha} p(z)\right| \leq n|\alpha| \max _{|z|=1}|p(z)| \text {. }
$$


Inequality (1.10) becomes equality for $p(z)=a z^{n}, a \neq 0$.

Further, Aziz [1] extended inequality (1.2) to polar derivative.

Theorem E. If $p(z)$ is a polynomial of degree $n$ having no zero in the disc $|z|<1$, then for every real or complex number $\alpha$ with $|\alpha| \geq 1$,

$$
\max _{|z|=1}\left|D_{\alpha} p(z)\right| \leq \frac{n}{2}(|\alpha|+1) \max _{|z|=1}|p(z)| .
$$

The result is best possible and extremal polynomial is $p(z)=z^{n}+1$.

For the class of polynomials not vanishing in the $\operatorname{disc}|z|<k, k \geq 1$, Aziz [1] obtained the extension of Theorem A to polar derivative of $p(z)$.

Theorem F. If $p(z)$ is a polynomial of degree $n$ having no zero in the disc $|z|<k, k \geq 1$, then for every real or complex number $\alpha$ with $|\alpha| \geq 1$,

$$
\max _{|z|=1}\left|D_{\alpha} p(z)\right| \leq n\left(\frac{k+|\alpha|}{1+k}\right) \max _{|z|=1}|p(z)|
$$

The result is best possible and equality in (1.12) holds for the polynomial $p(z)=(z+k)^{n}$, with $\alpha \geq 1$.

In this paper, we extend both the Theorems B and C into polar derivative of a polynomial. More precisely, we prove

Theorem 1. If $p(z)$ is a polynomial of degree $n$ such that $p(z) \neq 0$ in $|z|<k, k \leq 1$, then for every real or complex number $\alpha$ with $|\alpha| \geq 1$,

$$
\max _{|z|=1}\left|D_{\alpha} p(z)\right| \leq n \frac{\left(k^{n}+|\alpha|\right)}{1+k^{n}} \max _{|z|=1}|p(z)|
$$

provided $\left|p^{\prime}(z)\right|$ and $\left|q^{\prime}(z)\right|$ attain their maxima at the same point on the circle $|z|=1$, where $q(z)=z^{n} \overline{p\left(\frac{1}{\bar{z}}\right)}$

Dividing both sides of (1.13) by $|\alpha|$ and making limit as $|\alpha| \rightarrow \infty$, we obtain inequality (1.6).

Next, we prove the polar derivative form of Theorem $\mathrm{C}$.

Theorem 2. If $p(z)$ is a polynomial of degree $n$ such that $p(z) \neq 0$ in $|z|<k, k \leq 1$, then for every real or complex number $\alpha$ with $|\alpha| \geq 1$,

$$
\max _{|z|=1}\left|D_{\alpha} p(z)\right| \leq \frac{n}{1+k^{n}}\left\{\left(k^{n}+|\alpha|\right) \max _{|z|=1}|p(z)|-(|\alpha|-1) \min _{|z|=k}|p(z)|\right\},
$$

provided $\left|p^{\prime}(z)\right|$ and $\left|q^{\prime}(z)\right|$ attain their maxima at the same point on the circle $|z|=1$, where $q(z)=z^{n} \overline{p\left(\frac{1}{\bar{z}}\right)}$.

Dividing both sides of (1.14) by $|\alpha|$ and making limit as $|\alpha| \rightarrow \infty$, we get inequality (1.7).

II LEMMA

The following lemma is needed for the proofs of the theorems.

Lemma 2.1. If $p(z)$ is a polynomial of degree $n$, then on $|z|=1$, 


$$
\left|p^{\prime}(z)\right|+\left|q^{\prime}(z)\right| \leq n \max _{|z|=1}|p(z)|
$$

where

$$
q(z)=z^{n} \overline{p\left(\frac{1}{\bar{z}}\right)}
$$

The above lemma is a special case of a result due to Govil and Rahman [7].

\section{PROOF OF THE THEOREM}

I.

Proof of Theorem 1. We omit the proof as it follows on the same lines as that of Theorem 2 by using Theorem B, instead of Theorem C.

Proof of Theorem 2. Let $q(z)=z^{n} \overline{p\left(\frac{1}{z}\right)}$. Then it is easy to verify that for $|z|=1$,

$$
\left|q^{\prime}(z)\right|=\left|n p(z)-z p^{\prime}(z)\right| \text {. }
$$

Now, for every real or complex number $\alpha$, the polar derivative of $p(z)$ with respect to $\alpha$ is

$$
D_{\alpha} p(z)=n p(z)+(\alpha-z) p^{\prime}(z) \text {. }
$$

This implies for $|z|=1$,

$$
\begin{aligned}
\left|D_{\alpha} p(z)\right| \leq\left|n p(z)-z p^{\prime}(z)\right|+|\alpha|\left|p^{\prime}(z)\right| \\
\quad=\left|q^{\prime}(z)\right|+|\alpha|\left|p^{\prime}(z)\right| \quad(\text { by (3.1)) } \\
=\left|q^{\prime}(z)\right|+\left|p^{\prime}(z)\right|-\left|p^{\prime}(z)\right|+|\alpha|\left|p^{\prime}(z)\right| . \\
\leq n \max _{|z|=1}|p(z)|+(|\alpha|-1)\left|p^{\prime}(z)\right| \text { (Lemma 2.1) }
\end{aligned}
$$

Since $|\alpha| \geq 1$, the above inequality when combined with inequality (1.7) of Theorem C gives

$$
\begin{gathered}
\max _{|z|=1}\left|D_{\alpha} p(z)\right| \leq n \max _{|z|=1}|p(z)|+(|\alpha|-1)\left\{\frac{n}{1+k^{n}}\left(\max _{|z|=1}|p(z)|-\min _{|z|=k}|p(z)|\right)\right\} \\
=\frac{n}{1+k^{n}}\left\{\left(k^{n}+|\alpha|\right) \max _{|z|=1}|p(z)|-(|\alpha|-1) \min _{|z|=k}|p(z)|\right\} .
\end{gathered}
$$

This completes the proof of Theorem 2.

\section{REFERENCES}

A. Aziz, "Inequalities for the polar derivative of a polynomial," J. Approx. theory, vol. 55, pp. b183-193, 1988.

A. Aziz and Q.G. Mohammad, " Simple proof of a Theorem of Erdös and Lax, " Proc.

1. Amer Math. Soc., vol. 80, pp. 119-122, 1980.

2. Aziz and Rather, “ $L^{q}$ Inequalities for polynomials,” Math. Ineq. Appl., vol. 1, pp. 177-191, 1998.

A. Aziz and W.M. Shah, "Inequalities for the polar derivative of a polynomial," Indian J.

3. Pure and Appl. Math. Vol. 29(2), pp. 163-173, 1998.

4. N.G. de-Bruijn, " Inequalities concerning polynomials in the complex domain, Nederl.

5. Akad. Wetench. Proc. Ser. A, vol. 50(1947), pp. 1265-1272, 1947, Indag. Math., vol. 9, pp. 591-598, 1947.

6. N.K. Govil, “'On the Theorem of S. Bernstein," Proc. Nat. Acad. Sci., vol. 50, pp. 50-52,1980.

7. N.K. Govil and Q.I. Rahman, "Functions of exponential type not vanishing in a half-plane and related polynomials," Trans. Amer. Math. Soc., vol. 137, pp. 501-517, 1969.

8. P.D. Lax, " Proof of a conjecture of P. Erdös on the derivative of a polynomial, " Bull. Amer. Math. Soc., vol. 50, pp. 509-513, 1944. 
9. M.A. Malik, " On the derivative of a polynomial, " J. London Math. Soc., vol. 1, pp. 57-60, 1969.

10. G.V. Milovanovic, D.S. Mitrinovic and Th. M. Rassias, " Topics in polynomials, Extremal properties, Inequalities, Zeros, " World Scientific Publishing Co., Singapore, 1994.

11. C. Schaeffer, "Inequalities of A. Markoff and S. N. Bernstein for polynomials and related functions, " Bull, Amer. Math. Soc., pp. 565- 579, 1941. 